\title{
Effective rates of convergence for Lipschitzian pseudocontractive mappings in general Banach spaces*
}

\author{
Daniel Körnlein and Ulrich Kohlenbach ${ }^{\dagger}$ \\ Department of Mathematics \\ Technische Universität Darmstadt \\ Schlossgartenstraße 7, 64289 Darmstadt, Germany
}

May 19, 2011

\begin{abstract}
This paper gives an explicit and effective rate of convergence for an asymptotic regularity result $\left\|T x_{n}-x_{n}\right\| \rightarrow 0$ due to Chidume and Zegeye in 2004 where $\left(x_{n}\right)$ is a certain pertubated Krasnoselski-Mann iteration schema for Lipschitz pseudocontractive self-mappings $T$ of closed and convex subsets of a real Banach space. We also give a qualitative strengthening of the theorem by Chidume and Zegeye by weakening the assumption of the existence of a fixed point. For the bounded case, our bound is polynomial in the data involved.
\end{abstract}

\section{Introduction}

A fundamental theorem in the early stages of metric fixed point theory is the following one by Krasnoselski which, apart from showing the existence of at least one fixed point, also provides a sequence approximating one of these fixed points.

Theorem (Krasnoselski) ( [23]). Let $K$ be a non-empty closed, convex and bounded subset of a uniformly convex Banach space $X$ and let $T$ be a nonexpansive mapping of $K$ into a compact subset of $K$. Then for every $x_{0} \in K$, the sequence

$$
x_{k+1}:=\frac{x_{k}+T x_{k}}{2}
$$

converges strongly to a fixed point $z \in K$ of $T$.

Finding a uniform rate of convergence for the Picard iteration of $T$ (depending on the starting point $x_{0}$ and the mapping or, in fact, just a bound on its initial displacement $\left.\left\|x_{0}-T\left(x_{0}\right)\right\|\right)$ of strict contractions is trivial as the Banach fixed point theorem already provides this. The second author has shown in [16] that there is no effective procedure to compute a rate of convergence uniformly dependent on the data $x_{0}$ and $T$ for the above so-called Krasnoselski iteration where $T$ is nonexpansive.

In [16] it is also shown that one can, however, extract from Krasnoselski's proof a uniform bound for the asymptotic regularity of $x_{k}$, i.e., for $\left\|T x_{k}-x_{k}\right\| \rightarrow 0$, which only depends on the modulus of (uniform) convexity and the diameter of the set $K$ (without any compactness condition needed). This was further generalized in [18] to a quantitative version of a theorem due to Groetsch [11] (see also [30]) for the Krasnoselski-Mann iterations

$$
x_{n+1}:=\left(1-c_{n}\right) x_{n}+c_{n} T x_{n}
$$

\footnotetext{
*The results of this paper are (in a somewhat improved form) from the Bachelor Thesis of the 1st author [15] written under the supervision of the 2nd author.

${ }^{\dagger}$ The 2nd author has been supported by the German Science Foundation (DFG Project KO 1737/5-1).
} 
for $\left(c_{n}\right)$ in $[0,1]$ with $\sum c_{n}\left(1-c_{n}\right)=\infty$.

In a landmark result, Ishikawa showed in 1976 [13] that the assumption of uniform convexity was superfluous even in Groetsch' theorem under a somewhat stronger condition on $\left(c_{n}\right)$ :

Theorem (Ishikawa) ( [13]). Let $K$ be a nonempty and convex subset of a Banach space $X$ and let $T: K \rightarrow K$ be a nonexpansive mapping. For $x_{0} \in K$ define the sequence $\left(x_{n}\right)$ by

$$
x_{n+1}:=\left(1-c_{n}\right) x_{n}+c_{n} T x_{n},
$$

where $\left(c_{n}\right)$ is a real sequence in $[0, b)$ for some $b \in(0,1)$ with $\sum_{n=0}^{\infty} c_{n}=\infty$. If $\left(x_{n}\right)$ is bounded, then $\lim _{n \rightarrow \infty}\left\|T x_{n}-x_{n}\right\|=0$.

Under the assumption that $K$ is compact, the sequence $\left(x_{n}\right)$ converges strongly to a fixed point of $T$. In [17] an effective rate of convergence for $\left(\left\|T x_{n}-x_{n}\right\|\right)$ was extracted from the proof of a more general theorem due to Borwein, Reich and Shafrir (see [2]). In [21] the result was even proved in hyperbolic space. For the special case of constant $\lambda_{n}:=\lambda \in(0,1)$ an optimal quadratic rate of convergence was obtained by Baillon and Bruck (see [1]).

In 1967, Browder introduced an important generalization of the class of nonexpansive, namely pseudocontractive mappings defined by

$$
\forall u, v \in K \forall \lambda>1((\lambda-1)\|u-v\| \leq\|(\lambda I-T)(u)-(\lambda I-T)(v)\|),
$$

where $I$ denotes the identity mapping.

Apart from being a generalization of nonexpansive mappings, the pseudocontractive mappings are also closely related to accretive operators, where an operator $A$ is called accretive if for every $u, v \in K$ and for all $s>0$,

$$
\|u-v\| \leq\|u-v+s(A u-A v)\| .
$$

Observe that $T$ is pseudocontractive if and only if $I-T$ is accretive. Therefore, any fixed point of $T$ is a root of $I-T$. Hence, pseudocontractive mappings have received considerable attention over the past 15 years.

According to Kato [14], there is also a different characterization for pseudocontractions using the dual space $X^{*}$ of the Banach space $X$ and the normalized duality mapping, which is defined by:

Definition 1. Let $X$ be a real Banach space with dual $X^{*}$. The normalized duality mapping $J: X \rightarrow 2^{X^{*}}$ is defined by

$$
J x:=\left\{f^{*} \in X^{*}:\left\langle x, f^{*}\right\rangle=\|x\|^{2}=\left\|f^{*}\right\|^{2}\right\}
$$

As a consequence of the Hahn-Banach Theorem, $J x$ is nonempty for all $x \in X$. We now state the characterization of pseudocontractive mappings due to Kato [14]: A mapping $T: U \rightarrow X$ is pseudocontractive if and only if for all $u, v \in U$ there exists a $j \in J(u-v)$ such that

$$
\langle T u-T v, j\rangle \leq\|u-v\|^{2} .
$$

Until 2000, it has been an open problem whether the Krasnoselski-Mann iteration could be used to approximate fixed points for Lipschitzian pseudocontractive mappings. In 2000, Mutangadura and Chidume [7] refuted this by giving an example of a Lipschitzian pseudocontractive mapping from the unit circle of the two-dimensional Euclidean space into itself for which $\lim \left\|T x_{n}-x_{n}\right\| \rightarrow 0$ does not hold.

In 1974, Ishikawa [12] published an iteration sequence which converges strongly to a fixed point of Lipschitzian pseudocontractive mappings $T$, but only on compact and convex subsets of Hilbert spaces: 
Theorem (Ishikawa) ( [12]). If $K$ is a compact and convex subset of a Hilbert space $H, T$ : $K \rightarrow K$ is a Lipschitz pseudocontractive mapping and $x_{0}$ is any point of $K$, then the sequence $\left(x_{n}\right)$ converges strongly to a fixed point of $T$, where $\left(x_{n}\right)$ is defined iteratively for each $n \in \mathbb{N}$ by

$$
\left\{\begin{aligned}
y_{n} & :=\left(1-\beta_{n}\right) x_{n}+\beta_{n} T x_{n}, \\
x_{n+1} & :=(1-\alpha) x_{n}+\alpha_{n} T y_{n},
\end{aligned}\right.
$$

where $\left(\alpha_{n}\right)$ and $\left(\beta_{n}\right)$ are sequences of real numbers which satisfy the following conditions:

(i) $0 \leq \alpha_{n} \leq b_{n}<1$,

(ii) $\lim _{n \rightarrow \infty} \beta_{n}=0$,

(iii) $\sum_{n=0}^{\infty} \alpha_{n} \beta_{n}=\infty$

It is still an open problem whether the Ishikawa iteration, i.e., the one presented in the above theorem, extends to more general spaces.

In 2004, Chidume and Zegeye [8] published a perturbation of the iteration method provided by (1) for which $\left\|T x_{n}-x_{n}\right\| \rightarrow 0$ holds for the more general case of Lipschitz pseudocontractive mappings. For this result, no compactness assumption is needed. Naturally, simply dropping this hypothesis completely would make the result false. Simply take $X:=K:=\mathbb{R}$ and $T: x \mapsto x+1$. Being nonexpansive, $T$ is Lipschitz continuous and pseudocontractive, but $\|T x-x\|=1$ for all $x \in \mathbb{R}$. Thus, one of two requirements should be added (here $F(T)$ denotes the fixed point set of $T):$

(i) $F(T) \neq \emptyset$, or

(ii) $K$ is bounded.

While the first condition is the one required in [8] (Theorem 3.1), the second is required in Remark 3.6 of the same paper.

Let us emphasize that the class of Lipschitz pseudocontractions not only properly contains the class of nonexpansive mappings but also the important class of strictly pseudo-contractive mappings in the sense of Browder and Petryshyn [4] (see [6] for a detailed discussion; the fact that strict pseudocontractions are Lipschitzean seems to be due to [26]). In [4] they proved a fixed point theorem for this class (see [4], Theorem 12) as well as - under a Leray-Schauder condition several such theorems for Lipschitz pseudocontractions (see [4], Theorems 14 and 15).

Theorem (Chidume, Zegeye) ( [8]). Let $K$ be a nonempty, closed and convex subset of a real Banach space $X$. Let $T: K \rightarrow K$ be a Lipschitz pseudocontractive mapping with Lipschitz constant $L \geq 0$ such that $F(T) \neq \emptyset$. Let a sequence $\left(x_{n}\right)$ be generated from an arbitrary $x_{1} \in K$ by

$$
x_{n+1}:=\left(1-\lambda_{n}\right) x_{n}+\lambda_{n} T x_{n}-\lambda_{n} \theta_{n}\left(x_{n}-x_{1}\right),
$$

for all $n \in \mathbb{N}$, where $\left(\lambda_{n}\right)$ and $\left(\theta_{n}\right)$ are sequences of real numbers in $(0,1]$ satisfying the following conditions:
(i) $\lim _{n \rightarrow \infty} \theta_{n}=0$
(ii) $\sum_{n=1}^{\infty} \lambda_{n} \theta_{n}=\infty$,
(iv) $\lim _{n \rightarrow \infty} \frac{\left(\frac{\theta_{n-1}}{\theta_{n}}-1\right)}{\lambda_{n} \theta_{n}}=0$,
(iii) $\lim _{n \rightarrow \infty} \frac{\lambda_{n}}{\theta_{n}}=0$,
(v) $\lambda_{n}\left(1+\theta_{n}\right) \leq 1$.

Then $\left\|x_{n}-T x_{n}\right\| \rightarrow 0$ as $n \rightarrow \infty$. 
The iteration scheme (2) was first considered by Bruck [5] in the context of Hilbert spaces and by Reich [29] for the class of uniformly convex Banach spaces (see also [28,31]).

The main result in this paper (Theorem 1 in Section 4) gives an explicit rate of convergence for the above theorem by Chidume and Zegeye. This rate depends on given rates of convergence and divergence ('moduli') $)^{1}$ corresponding to the conditions $(i)-(i v)$ on $\left(\theta_{n}\right)$ and $\left(\lambda_{n}\right)$ as well as an upper bound $b$ of $\left\|x_{1}-p\right\|$ where $p$ is a fixed point of $T$, the error and a Lipschitz constant of $T$. However the bound does not depend on $X, K, x_{1}$ and $T$. In fact the assumption of the existence of a fixed point of $T$ can be weakened to the existence of approximate fixed points in some $b$-ball around $x_{1}$.

For $\varepsilon>0$, we call $p_{\varepsilon}$ an ' $\varepsilon$ - approximate fixed point' (or simply ' $\varepsilon$-fixed point') of $T$ if $\left\|p_{\varepsilon}-T\left(p_{\varepsilon}\right)\right\|<$ $\varepsilon$. We say that $K$ has the 'approximate fixed point property' for some class of self-mappings $T: K \rightarrow K$ provided that each such $T$ has $\varepsilon$-fixed points in $K$ for each $\varepsilon>0$.

Under the additional assumption on $X$ having a Gâteaux differentiable norm and on $K$ having the fixed point property for nonexpansive self-mappings, Chidume and Zegeye also show the strong convergence of their iteration to a fixed point of $T$. It remains for a future research to extract from the proof an effective rate of metastability for this result.

\section{Logical analysis of proofs in nonlinear analysis}

This section is not needed for the proof of the main result of this paper but rather explains the broader logical context in which that proof was obtained. Going back to pioneering work of G. Kreisel in the 50's but having been taking up more systematically and in the context of nonlinear analysis only during the past decade, a general program of analyzing proofs using tools from logic (proof theory) emerged which has the aim to extract explicit effective and highly uniform bounds from given proofs (see [20] for a recent book treatment of that). In particular, general logical 'metatheorems' were developed that guarantee for large classes of (even highly ineffective) proofs in abstract nonlinear functional analysis the extractability of such bounds (see, for example, $[9,10,19,20])$. These metatheorems have meanwhile been applied to numerous prima facie ineffective proofs of asymptotic regularity statements

$$
(*)\left\|x_{n}-T x_{n}\right\| \rightarrow 0
$$

of certain iteration sequences $\left(x_{n}\right)$ in metric fixed point theory based on general classes of mappings $T$ in Hilbert, Banach, hyperbolic or CAT(0)-spaces among others. The metatheorems guarantee that the extracted bound (besides being computable) is guaranteed to be essentially independent of the underlying space, the operator $T$ and the starting point $x_{1}$ of the iteration except for some local metric bound $b$ namely on $\left\|T x_{1}-x_{1}\right\|,\left\|x_{1}\right\|$ and on $\left\|x_{1}-p\right\|$ if the existence of some fixed point $p$ of $T$ is assumed. If the proof essentially proceeds by contradiction, then the statement to be proved needs the following logical form $\forall x \exists y A_{q f}(x, y)$, where $A_{q f}$ (essentially) is a quantifier-free formula. In $(*)$ above, which formalizes as

$$
(* *) \forall k \in \mathbb{N} \exists n \in \mathbb{N} \forall m \geq n\left(\left\|T x_{m}-x_{m}\right\| \leq 2^{-k}\right),
$$

this is not the case. However, in many cases the monotonicity of the sequence allows one to drop the innermost universal quantifier $\forall m \geq n$ without changing the statement. If this is not possible, then one in general has to replace $(* *)$ by the (ineffectively equivalent) so-called Herbrand normal form of $(* *)$

$$
\forall k \in \mathbb{N} \forall g: \mathbb{N} \rightarrow \mathbb{N} \exists n \in \mathbb{N} \forall m \in[n, n+g(n)]\left(\left\|T x_{m}-x_{m}\right\| \leq 2^{-k}\right)
$$

which recently has been rediscovered by T. Tao [32] under the name of 'metastability'. For a recent application of this in metric fixed point theory see, for instance, [22]. In this paper we surprisingly

\footnotetext{
${ }^{1}$ These moduli provide quantitative versions of the convergence/divergence conditions $(i)$ - $(i v)$. In logic such quantitative enrichments are called 'Skolem functions'. In the final section we will show how to compute such moduli for concrete sequences $\left(\lambda_{n}\right)$ and $\left(\theta_{n}\right)$.
} 
obtain a full rate of convergence (i.e. an effective bound on $\exists n \in \mathbb{N}$ in $(* *)$ ) despite of the fact that monotonicity of the iteration sequences apparently does not hold. This is due to the fact that in the course of the extraction the proof from [8] turns out to be essentially constructively reformulatable. In such cases one can omit a logical step necessary in the ineffective case: the so-called negative translation of Gödel. As a consequence one can directly apply proof interpretations such as the monotone modified realizability or monotone functional interpretation (see $[9,20])$. These are proof-theoretic techniques which extract uniform bounds $\Phi$

$$
\forall k \in \mathbb{N} \forall m \geq \Phi(k)\left(\left\|T x_{m}-x_{m}\right\| \leq 2^{-k}\right)
$$

from proofs of statements $(* *)$. 'Uniform' here means that the bound - in addition to $k$ - only depends on a bound $b \geq\left\|x_{1}\right\|,\left\|T x_{1}-x_{1}\right\|,\left\|p-x_{1}\right\|$ for some $p \in F(T)$, a Lipschitz constant $L$ for $T$ and the aforementioned quantitative moduli $R_{1}, \ldots, R_{4}$. Such moduli are asked for by the proof interpretations just mentioned to make the conditions $(i)-(i v)$ on $\left(\lambda_{n}\right),\left(\theta_{n}\right)$ purely universal (see section 4 below). The universal quantification over $\left(\theta_{n}\right)$ and $\left(\lambda_{n}\right)$ can be taken in the whole compact Polish space ${ }^{2}[0,1]^{\mathbb{N}}$. It follows (again by the aforementioned logical metatheorems) that the bound $\Phi$ does not depend on these sequences themselves but only on their moduli $R_{1}, \ldots R_{4}$.

Condition $(v)$ is already purely universal (and hence left unchanged by the logical interpretation) as are the conditions on $T$ being $L$-Lipschitz and pseudocontractive and $p$ being a fixed point of $T$. The monotone proof interpretations mentioned above also require a so-called majorizing function $T^{*}: \mathbb{N} \rightarrow \mathbb{N}$ for $T$ to be available as an additional argument of $\Phi$, i.e., a nondecreasing function $T^{*}$ such that

$$
\forall n \in \mathbb{N} \forall x \in K\left(n \geq\|x\| \rightarrow T^{*}(n) \geq\|T(x)\|\right)
$$

However, under the assumptions above one can simply take, e.g., $T^{*}(n):=L(n+b)+2 b$ since

$$
n \geq\|x\| \rightarrow\|T x\| \leq\left\|T x-T x_{1}\right\|+\left\|T x_{1}-x_{1}\right\|+\left\|x_{1}\right\| \leq L\left\|x-x_{1}\right\|+2 b \leq L(n+b)+2 b .
$$

Hence we do not have to add $T^{*}$ as an additional input to $\Phi$.

Chidume and Zegeye assume that $T$ has a fixed point $p \in K$, i.e., that $\forall l \in \mathbb{N}\left(\|T p-p\| \leq 2^{-l}\right)$. First we note that it is sufficient to impose a bound $b$ on $\left\|x_{1}\right\|$ and $\left\|x_{1}-p\right\|$ which is needed to compute a majorant $T^{*}$ since

$$
\left\|T x_{1}-x_{1}\right\| \leq\left\|T x_{1}-T p\right\|+\left\|p-x_{1}\right\| \leq L b+b .
$$

Now consider the implication

$$
\forall l \in \mathbb{N}\left(\|T p-p\| \leq 2^{-l}\right) \rightarrow \forall k \in \mathbb{N} \exists n \in \mathbb{N} \forall m \geq n\left(\left\|T x_{m}-x_{m}\right\| \leq 2^{-k}\right)
$$

In contrast to the monotone modified realizability interpretation, the monotone functional interpretation not only extracts a bound $\Phi$ on ' $\exists n \in \mathbb{N}$ ' but also a bound on the negatively occurring universal quantifier ' $\forall l \in \mathbb{N}$ ' in the premise that, however, has both $k$ and $m$ as arguments (see [20] for more details):

$$
\forall k, m \in \mathbb{N}\left(\|T p-p\| \leq 2^{-f(k, m)}\right) \rightarrow\left(m \geq \Phi(k) \rightarrow\left\|T x_{m}-x_{m}\right\| \leq 2^{-k}\right),
$$

where $f$ only depends on $b$ and $L$ but not on $p$. Now suppose that in some fixed ball around $x_{1}$ arbitrarily good approximate fixed points of $T$ exist and assume that $b$ is also a bound on the radius of this ball. If we now pick for given $k$ and $m \geq \Phi(k)$ a $2^{-f(k, m)}$-good such approximate fixed point as $p$, then we get $\left\|T x_{m}-x_{m}\right\| \leq 2^{-k}$. Since we can do this for every $k, m$ we obtain

$$
\forall k \in \mathbb{N} \forall m \geq \Phi(k)\left(\left\|T x_{m}-x_{m}\right\| \leq 2^{-k}\right)
$$

\footnotetext{
${ }^{2}$ The theorem by Chidume and Zegeye [8] assumes that $\theta_{n}, \lambda_{n} \in(0,1]$ but the proof can be rewritten in such a way that the case that $\theta_{n}=0$ or $\lambda_{n}=0$ for certain $n$ is allowed (see [15] where this is carried out). Since, however, things get considerably simpler under the original assumption $\theta_{n}, \lambda_{n}>0$ we confine ourselves here to this one.
} 
and so, in particular, that $\lim \left\|T x_{n}-x_{n}\right\|=0$ (together with the rate of convergence $\Phi$ ) under the weaker assumption that in some ball around $x_{1}$ the mapping $T$ has arbitrarily good approximate fixed points. Using implicitly this approach, gives in our main theorem in section 4 below not only the effective bound $\Phi$ but also a verification of this bound under that weakened hypothesis on $T$. Bounding $\left\|T x_{1}-x_{1}\right\|$ in terms of $b$ and $L$ still works when $p$ is just some $2^{-l}$-fixed point using now $L b+b+1$ as upper bound. The actual bound extracted in section 4 even only uses that $b \geq\left\|x_{1}-p\right\|$ but no upper bound on $\left\|x_{1}\right\|$ itself. This is due to the fact that the whole proof never touches any points outside of $K$ hence one can take $x_{1}$ as a reference point w.r.t. which we majorize distances (see [20], in particular pp. 410-411).

\section{Technical Lemmas}

The following lemma is a quantitative version of a lemma due to Moore and Nnoli [24]. In the following, $\mathbb{R}_{+}^{*}$ denotes the set of strictly positive real numbers.

Lemma 1. Let $\psi:(0, \infty) \rightarrow(0, \infty)$ be an increasing function (formally extended to 0 by $\psi(0):=0$ ) and let $\left(a_{n}\right),\left(b_{n}\right)$ and $\left(c_{n}\right)$ be sequences of real nonnegative numbers with modulus $N_{1}:(0, \infty) \rightarrow \mathbb{N}$ such that: ${ }^{3}$

$$
\forall \varepsilon>0 \forall n \geq N_{1}(\varepsilon)\left(c_{n} \leq b_{n} \varepsilon\right) .
$$

Moreover, let $\sum_{n=1}^{\infty} b_{n}=\infty$ with rate of divergence $N_{2}:(0, \infty) \rightarrow \mathbb{N}$,

$$
\forall x \in(0, \infty)\left(\sum_{i=1}^{N_{2}(x)} b_{i}>x\right) .
$$

If for all $n \geq 0$

$$
a_{n+1}^{2} \leq a_{n}^{2}-b_{n} \psi\left(a_{n+1}\right)+c_{n}
$$

then we get a rate of convergence for $\left(a_{n}\right)$ :

$$
\forall \varepsilon>0 \forall n \geq \Phi\left(N_{1}, N_{2}, \varepsilon\right)\left(a_{n}<\varepsilon\right),
$$

where $\Phi: \mathbb{N}^{\mathbb{R}_{+}^{*}} \times \mathbb{N}^{\mathbb{R}_{+}^{*}} \times \mathbb{R}_{+}^{*} \rightarrow \mathbb{N}$, with

$$
\begin{aligned}
& \Phi\left(N_{1}, N_{2}, \varepsilon\right)=N_{2}(C)+1, \\
& C \geq \frac{2 a_{\tilde{N}}^{2}}{\psi(\varepsilon)}+\sum_{n=1}^{\tilde{N}-1} b_{n}, \\
& \tilde{N}=N_{1}\left(\frac{\psi(\varepsilon)}{2}\right) .
\end{aligned}
$$

Proof. Let $\varepsilon>0$ be given. We split the proof into two claims:

Claim 1: $\exists n \in\left[\tilde{N}, N_{2}(C)+1\right]\left(a_{n}<\varepsilon\right)$

Proof. Assume on the contrary that

$\forall n \in\left[\tilde{N}, N_{2}(C)+1\right]\left(a_{n} \geq \varepsilon\right)$. We first remark that the above interval is non-trivial since for $\tilde{N} \geq N_{2}(C)+1$, we would get

$$
\sum_{n=1}^{N_{2}(C)} b_{n} \leq \sum_{n=1}^{\tilde{N}-1} b_{n} \leq \sum_{n=1}^{\tilde{N}-1} b_{n}+\frac{2 a_{\tilde{N}}^{2}}{\psi(\varepsilon)} \leq C,
$$

which is a contradiction to assumption (4), i.e., to the rate of divergence of $\sum b_{n}$. So from this we must have $\tilde{N}<N_{2}(C)+1$.

\footnotetext{
${ }^{3}$ For strictly positive $b_{n},(3)$ just expresses that $c_{n} / b_{n}$ tends to 0 with rate of convergence $N_{1}$.
} 
By assumption (3), we get

$$
\forall n \geq \tilde{N}\left(c_{n} b_{n}^{-1} \leq \frac{1}{2} \psi(\varepsilon)\right),
$$

and so for all $n \in\left[\tilde{N}, N_{2}(C)\right]$ it follows that

$$
\begin{aligned}
a_{n+1}^{2} & \leq a_{n}^{2}-b_{n} \psi\left(a_{n+1}\right)+c_{n} \\
& \leq a_{n}^{2}-b_{n} \psi(\varepsilon)+\frac{1}{2} \psi(\varepsilon) b_{n} \\
& =a_{n}^{2}-\frac{1}{2} \psi(\varepsilon) b_{n} .
\end{aligned}
$$

Therefore

$$
\frac{1}{2} \psi(\varepsilon) b_{n} \leq a_{n}^{2}-a_{n+1}^{2} .
$$

Since this holds for all $n \in\left[\tilde{N}, N_{2}(C)\right]$, summing up yields

$$
\frac{1}{2} \psi(\varepsilon) \sum_{n=\tilde{N}}^{N_{2}(C)} b_{n} \leq a_{\tilde{N}}^{2}-a_{N_{2}(C)}^{2} \leq a_{\tilde{N}}^{2} .
$$

Hence after adding $\sum_{n=1}^{\tilde{N}-1} b_{n}$ to $(6)$ we get

$$
\sum_{n=1}^{N_{2}(C)} b_{n} \leq \sum_{n=1}^{\tilde{N}-1} b_{n}+\frac{2 a_{\tilde{N}}^{2}}{\psi(\varepsilon)} \leq C,
$$

which is again a contradiction to (4). This proves Claim 1.

Then for some $n_{0} \in\left[N_{1}\left(\frac{\psi(\varepsilon)}{2}\right), N_{2}(C)+1\right]$ we know that $a_{n_{0}}<\varepsilon$. We now prove the following claim, which will conclude our result.

Claim 2: $\forall l\left(a_{n_{0}+l}<\varepsilon\right)$.

Proof. By induction on $l$. For $l=0$ this is clear. We prove the induction step by contradiction. So assume that for some $l \geq 0$, we have $a_{n_{0}+l}<\varepsilon$ but $a_{n_{0}+l+1} \geq \varepsilon$. Using (3) again we get

$$
\begin{aligned}
\varepsilon^{2} & \leq a_{n_{0}+l+1}^{2} \\
& \leq a_{n_{0}+l}^{2}-b_{n_{0}+l} \psi\left(a_{n_{0}+l+1}\right)+c_{n_{0}+l} \\
& \leq a_{n_{0}+l}^{2}-b_{n_{0}+l} \psi(\varepsilon)+b_{n_{0}+l} \frac{\psi(\varepsilon)}{2} \\
& <\varepsilon^{2}-\frac{b_{n_{0}+l}}{2} \psi(\varepsilon) \\
& \leq \varepsilon^{2}
\end{aligned}
$$

which is a contradiction.

In the proof of our main Theorem 1, we will need a quantitative form of Proposition 2(iv) from Morales and Jung [25].

Lemma 2. Let $X$ be a Banach space and let $K$ be a closed and convex subset of $X$. Suppose that $T: K \rightarrow K$ is a pseudocontractive mapping such that for each $x_{1} \in K$, the equation

$$
x=t T x+(1-t) x_{1}
$$

has a solution $y_{t} \in K$ for every $t \in(0,1)$. If $\left\|T x_{\varepsilon}-x_{\varepsilon}\right\| \leq \varepsilon$, then

$$
\left\|y_{t}-x_{\varepsilon}\right\| \leq\left\|x_{1}-x_{\varepsilon}\right\|+\frac{t \varepsilon}{1-t}
$$

for all $t \in(0,1)$ 
Proof. From the pseudocontractiveness, there exists a $j \in J\left(y_{t}-x_{\varepsilon}\right)$ such that

$$
\begin{aligned}
\left\langle y_{t}-x_{\varepsilon}, j\right\rangle & =\left\langle t T y_{t}+(1-t) x_{1}-x_{\varepsilon}, j\right\rangle \\
& =t\left\langle T y_{t}-T x_{\varepsilon}, j\right\rangle+t\left\langle T x_{\varepsilon}-x_{\varepsilon}, j\right\rangle+(1-t)\left\langle x_{1}-x_{\varepsilon}, j\right\rangle \\
& \leq \underbrace{t\left\langle y_{t}-x_{\varepsilon}, j\right\rangle}_{=t\left\|y_{t}-x_{\varepsilon}\right\|^{2}=t\|j\|^{2}}+t\left\|T x_{\varepsilon}-x_{\varepsilon}\right\| \cdot\|j\|+(1-t)\left\|x_{1}-x_{\varepsilon}\right\| \cdot\|j\| .
\end{aligned}
$$

Now, if $\|j\|=0$, we also have that $\left\|y_{t}-x_{\varepsilon}\right\|=0$ and the claim becomes trivial. Otherwise, dividing by $\|j\|$ implies that

$$
(1-t)\left\|y_{t}-x_{\varepsilon}\right\| \leq(1-t)\left\|x_{1}-x_{\varepsilon}\right\|+t\left\|T x_{\varepsilon}-x_{\varepsilon}\right\| .
$$

Hence the claim follows from $\left\|T x_{\varepsilon}-x_{\varepsilon}\right\| \leq \varepsilon$.

The next lemma is an improved version of the following inequality

$$
\left\|y_{n-1}-y_{n}\right\| \leq\left|\frac{\theta_{n-1}}{\theta_{n}}-1\right|\left(\left\|y_{n-1}\right\|+\left\|x_{1}\right\|\right)
$$

used by Chidume and Zegeye. Since Chidume and Zegeye are only interested in qualitative results, they do not need the stronger version.

Lemma 3. Let $K$ be a nonempty subset of a real Banach space $X$. Let $T: K \rightarrow K$ be a Lipschitz pseudocontractive mapping and let $\left(\theta_{n}\right) \subset(0,1]$ be a real sequence. Denote $t_{n}:=\frac{1}{1+\theta_{n}}$. Let $\left(y_{n}\right)$ be a sequence in $K$ defined by $y_{n}=t_{n} T y_{n}+\left(1-t_{n}\right) x_{1}$. Then

$$
\left\|y_{n-1}-y_{n}\right\| \leq\left|\frac{\theta_{n-1}}{\theta_{n}}-1\right|\left\|y_{n-1}-x_{1}\right\| .
$$

for all $n \in \mathbb{N}$.

Proof. From the definition of $y_{n}$ we immediately get

$$
\begin{aligned}
\left(1+\frac{1}{\theta_{n}}\right) y_{n} & =\left(1+\frac{1}{\theta_{n}}\right)\left(\frac{1}{1+\theta_{n}} T y_{n}+\frac{\theta_{n}}{1+\theta_{n}} x_{1}\right) \\
& =\frac{1+\theta_{n}}{\theta_{n}}\left(\frac{1}{1+\theta_{n}} T y_{n}+\frac{\theta_{n}}{1+\theta_{n}} x_{1}\right) \\
& =\frac{T y_{n}}{\theta_{n}}+x_{1} .
\end{aligned}
$$

Since $\left(1+\frac{1}{\theta_{n}}\right)=\frac{1+\theta_{n}}{\theta_{n}}=\frac{1}{t_{n} \theta_{n}}$, the pseudocontractivity of $T$ (applied to $\lambda:=1+\theta_{n}$ ) together with (7) implies

$$
\begin{aligned}
\left\|y_{n-1}-y_{n}\right\| & \leq\left\|y_{n-1}-y_{n}+\frac{1}{\theta_{n}}\left(y_{n-1}-T y_{n-1}-y_{n}+T y_{n}\right)\right\| \\
& =\left\|y_{n-1}\left(1+\frac{1}{\theta_{n}}\right)-y_{n}\left(1+\frac{1}{\theta_{n}}\right)-\frac{T y_{n-1}-T y_{n}}{\theta_{n}}\right\| \\
& =\left\|\frac{y_{n-1}}{t_{n} \theta_{n}}-\left(\frac{T y_{n}}{\theta_{n}}+x_{1}\right)-\frac{T y_{n-1}}{\theta_{n}}+\frac{T y_{n}}{\theta_{n}}\right\| \\
& =\left\|\frac{y_{n-1}}{t_{n} \theta_{n}}-\frac{T y_{n-1}}{\theta_{n}}-x_{1}\right\| .
\end{aligned}
$$

From $y_{n-1}=t_{n-1} T y_{n-1}+\left(1-t_{n-1}\right) x_{1}$ we obtain that $T y_{n-1}=\frac{y_{n-1}-\left(1-t_{n-1}\right) x_{1}}{t_{n-1}}$, hence

$$
\begin{aligned}
\left\|y_{n-1}-y_{n}\right\| & \leq\left\|\frac{y_{n-1}}{t_{n} \theta_{n}}-\frac{y_{n-1}-\left(1-t_{n-1}\right) x_{1}}{t_{n-1} \theta_{n}}-x_{1}\right\| \\
& =\left\|y_{n-1}\left(\frac{1}{t_{n} \theta_{n}}-\frac{1}{t_{n-1} \theta_{n}}\right)+x_{1}\left(\frac{1-t_{n-1}}{t_{n-1} \theta_{n}}-1\right)\right\| .
\end{aligned}
$$


Moreover

$$
\begin{aligned}
\frac{1}{t_{n} \theta_{n}}-\frac{1}{t_{n-1} \theta_{n}} & =\frac{1}{\theta_{n}}\left(\frac{1}{t_{n}}-\frac{1}{t_{n-1}}\right) \\
& =\frac{1}{\theta_{n}}\left(1+\theta_{n}-1-\theta_{n-1}\right) \\
& =1-\frac{\theta_{n-1}}{\theta_{n}}
\end{aligned}
$$

and

$$
\begin{aligned}
\frac{1-t_{n-1}}{t_{n-1} \theta_{n}}-1 & =\frac{1}{\theta_{n}}\left(\frac{1}{t_{n-1}}-1\right)-1 \\
& =\frac{1}{\theta_{n}}\left(1+\theta_{n-1}-1\right)-1 \\
& =\frac{\theta_{n-1}}{\theta_{n}}-1 .
\end{aligned}
$$

From (8), (9) and (10) we finally conclude

$$
\left\|y_{n}-y_{n-1}\right\| \leq\left|\frac{\theta_{n-1}}{\theta_{n}}-1\right| \cdot\left\|y_{n-1}-x_{1}\right\| .
$$

The proof in Chidume and Zegeye [8] also needs the following lemma due to W.V. Petryshyn

Lemma 4 (cf. [27]). Let $X$ be a real normed linear space and let $J$ be the normalized duality mapping on $X$. Then for any $x, y \in X$ and $j(x+y) \in J(x+y)$ the following inequality holds:

$$
\|x+y\|^{2} \leq\|x\|^{2}+2\langle y, j\rangle \text {. }
$$

\section{Main Results}

Let $\left(\lambda_{n}\right)$ and $\left(\theta_{n}\right)$ be real sequences in $(0,1]$ such that for all $n \in \mathbb{N}$ the following hold

(i) $\lim _{n \rightarrow \infty} \theta_{n}=0$,

(ii) $\sum_{n=1}^{\infty} \lambda_{n} \theta_{n}=\infty$,

(iii) $\forall \varepsilon>0 \exists m \in \mathbb{N} \forall n \geq m\left(\lambda_{n} \leq \theta_{n} \varepsilon\right)$,

(iv) $\forall \varepsilon>0 \exists m \in \mathbb{N} \forall n \geq m\left(\frac{\left|\frac{\theta_{n-1}}{\theta_{n}}-1\right|}{\lambda_{n} \theta_{n}} \leq \varepsilon\right)$.

(v) $\lambda_{n}\left(1+\theta_{n}\right) \leq 1$.

As in Lemma 1, we will also need the following rates of convergence and divergence $R_{i}:(0, \infty) \rightarrow \mathbb{N}$ such that

(i) $\forall \varepsilon>0 \forall n \geq R_{1}(\varepsilon)\left(\theta_{n} \leq \varepsilon\right)$,

(ii)' $\forall x \in(0, \infty)\left(\sum_{n=1}^{R_{2}(x)} \lambda_{n} \theta_{n} \geq x\right)$,

(iii)' $\forall \varepsilon>0 \forall n \geq R_{3}(\varepsilon)\left(\lambda_{n} \leq \theta_{n} \varepsilon\right)$, 
(iv)' $\forall \varepsilon>0 \forall n \geq R_{4}(\varepsilon)\left(\frac{\left|\frac{\theta_{n-1}}{\theta_{n}}-1\right|}{\lambda_{n} \theta_{n}} \leq \varepsilon\right)$.

Theorem 1. Let $K$ be a nonempty, closed and convex subset of a real Banach space $X$. Let $T: K \rightarrow K$ be a Lipschitzian pseudocontractive mapping with Lipschitz constant $L$ and for some $b>0$ assume that $T$ does possess arbitrarily good $\varepsilon$-fixed points $x_{\varepsilon} \in K$ with $\left\|x_{1}-x_{\varepsilon}\right\|<b$. Let $\left(x_{n}\right)$ be the sequence generated from an arbitrary $x_{1} \in K$ by

$$
x_{n+1}:=\left(1-\lambda_{n}\right) x_{n}+\lambda_{n} T x_{n}-\lambda_{n} \theta_{n}\left(x_{n}-x_{1}\right) .
$$

Given the rates of convergence/divergence $R_{i}: \mathbb{R} \rightarrow \mathbb{N}$ that satisfy (i)' to (iv)' above, we get

$$
\forall \varepsilon>0 \forall n \geq \Psi\left(b, L, R_{1}, R_{2}, R_{3}, R_{4}, \varepsilon\right)\left(\left\|x_{n}-T x_{n}\right\|<\varepsilon\right)
$$

where

$$
\Psi\left(b, L, R_{1}, R_{2}, R_{3}, R_{4}, \varepsilon\right)=\max \left\{N_{2}(C)+1, R_{1}\left(\frac{\varepsilon}{4 r}\right)+1\right\}
$$

and

$$
\begin{aligned}
& N_{1}(\varepsilon):=\max \left\{R_{3}\left(\frac{2 \varepsilon d}{3 r^{2}}\right), R_{4}\left(\sqrt{\frac{\varepsilon}{r^{2}}+\frac{9}{4}}-\frac{3}{2}\right)\right\} \\
& N_{2}(x):=R_{2}\left(\frac{x}{2}\right)+1 \\
& C:=\frac{18(1+L)^{2} r^{2}}{\varepsilon^{2}}+2\left(N_{1}\left(\frac{\varepsilon^{2}}{8(1+L)^{2}}\right)-1\right), \\
& r:=\max \left\{\frac{(2+L)^{R_{3}(d)}-1}{1+L} b, 2 b\right\}, \\
& d:=\frac{1}{2\left(\frac{5}{2}+L\right)(2+L)} .
\end{aligned}
$$

Remark 1. Note that the bound $\Psi$ does not depend on $X, K, T, x_{1}$ except for $b$ and the Lipschitz constant $L$.

Proof. Let $\varepsilon>0$ be given. For $\delta>0$, let $x_{\delta}$ be a $\delta$-fixed point of $T$ such that $\left\|x_{1}-x_{\delta}\right\|<b$, whose existence is guaranteed by the assumption. Let $c:=\frac{\left(2+L R^{R_{3}(d)}-1\right.}{1+L}$ and define

$$
\tilde{\delta}_{n, k}:=\left\{\begin{array}{cl}
2^{-k} c^{-1}, & \text { if } n \leq R_{3}(d) \\
\frac{2 \lambda_{n-1} \theta_{n-1}}{2+2 \lambda_{n-1}^{2}(2+L)} \cdot 2^{-k-1}, & \text { if } n>R_{3}(d)
\end{array} \text { and } \delta_{n, k}:=\min \left\{\tilde{\delta}_{i, k}: i \leq n\right\} .\right.
$$

We first show that $\left\|x_{n}-x_{\delta_{n, k}}\right\| \leq r+2^{-k}$ for all $n \leq R_{3}(d)$ and for any $k \in \mathbb{N}$. By the triangle inequality,

$$
\begin{aligned}
\left\|x_{n}-T x_{n}\right\| & \leq\left\|x_{n}-x_{\delta_{n, k}}\right\|+\left\|x_{\delta_{n, k}}-T x_{\delta_{n, k}}\right\|+\left\|T x_{\delta_{n, k}}-T x_{n}\right\| \\
& \leq\left\|x_{n}-x_{\delta_{n, k}}\right\|+\delta_{n, k}+\left\|T x_{\delta_{n, k}}-T x_{n}\right\| \\
& =(1+L)\left\|x_{n}-x_{\delta_{n, k}}\right\|+\delta_{n, k} .
\end{aligned}
$$

Moreover, since $\lambda_{n}, \theta_{n} \in(0,1]$ for all $n \geq 1$, we get

$$
\begin{aligned}
\left\|x_{n}-\lambda_{n} \theta_{n}\left(x_{n}-x_{1}\right)-x_{\delta_{n, k}}\right\| & =\left\|\left(1-\lambda_{n} \theta_{n}\right) x_{n}+\lambda_{n} \theta_{n} x_{1}-x_{\delta_{n, k}}\right\| \\
& \leq\left(1-\lambda_{n} \theta_{n}\right)\left\|x_{n}-x_{\delta_{n, k}}\right\|+\left\|\left(1-\lambda_{n} \theta_{n}\right) x_{\delta_{n, k}}+\lambda_{n} \theta_{n} x_{1}-x_{\delta_{n, k}}\right\| \\
& =\left(1-\lambda_{n} \theta_{n}\right)\left\|x_{n}-x_{\delta_{n, k}}\right\|+\lambda_{n} \theta_{n}\left\|x_{1}-x_{\delta_{n, k}}\right\| \\
& <\left\|x_{n}-x_{\delta_{n, k}}\right\|+\left\|x_{1}-x_{\delta_{n, k}}\right\| \\
& <\left\|x_{n}-x_{\delta_{n, k}}\right\|+b .
\end{aligned}
$$


From (12), (14) and the definition of $x_{n}$ we obtain for all $n, k$

$$
\begin{aligned}
\left\|x_{n+1}-x_{\delta_{n, k}}\right\| & =\left\|\left(1-\lambda_{n}\right) x_{n}+\lambda_{n} T x_{n}-\lambda_{n} \theta_{n}\left(x_{n}-x_{1}\right)-x_{\delta_{n, k}}\right\| \\
& \leq \lambda_{n}\left\|x_{n}-T x_{n}\right\|+\left\|x_{n}-\lambda_{n} \theta_{n}\left(x_{n}-x_{1}\right)-x_{\delta_{n, k}}\right\| \\
& <(1+L)\left\|x_{n}-x_{\delta_{n, k}}\right\|+\left\|x_{n}-x_{\delta_{n, k}}\right\|+b+\delta_{n, k} \\
& =(2+L)\left\|x_{n}-x_{\delta_{n, k}}\right\|+b+\delta_{n, k} .
\end{aligned}
$$

Since $\left\|x_{1}-x_{\delta_{n, k}}\right\|<b$ for all $n$ and $k$, it follows that for $n \leq R_{3}(d)$

$$
\begin{aligned}
\left\|x_{n}-x_{\delta_{n, k}}\right\| & <\sum_{i=0}^{n-1}(2+L)^{i}\left(b+2^{-k} c^{-1}\right) \\
& =\frac{(2+L)^{n}-1}{(2+L)-1}\left(b+2^{-k} c^{-1}\right) \\
& \leq \frac{(2+L)^{R_{3}(d)}-1}{1+L}\left(b+2^{-k} c^{-1}\right) \\
& =r+2^{-k} .
\end{aligned}
$$

Note that this estimate holds for any $\delta_{n, k}\left(=2^{-k} c^{-1}\right)$-fixed point $x_{\delta_{n, k}}$.

We now show by induction on $n \geq R_{3}(d)$ that $\left\|x_{n}-x_{\delta_{n, k}}\right\| \leq r+2^{-k}$ for all $k \in \mathbb{N}$ and for all $\delta_{n, k}$-fixed points $x_{\delta_{n, k}}$ of $T$ with $\left\|x_{1}-x_{\delta_{n, k}}\right\|<b$. For $n=R_{3}(d)$ this is trivial by (15). We show the induction step by contradiction. Assume $\left\|x_{n}-x_{\delta_{n, k}}\right\| \leq r+2^{-k}$ for any such $\delta_{n, k}$-fixed point $x_{\delta_{n, k}}$ but $\left\|x_{n+1}-x_{\delta_{n+1, k}}\right\|>r+2^{-k}$ for some $n \geq R_{3}(d)$ and for some $\delta_{n+1, k}$-fixed point $x_{\delta_{n+1, k}}$. Then using lemma 4 one shows exactly as in [8] that for all $j \in J\left(x_{n+1}-x_{\delta_{n+1, k}}\right)$

$$
\begin{aligned}
\left\|x_{n+1}-x_{\delta_{n+1, k}}\right\|^{2}= & \left\|x_{n}-x_{\delta_{n+1, k}}-\lambda_{n}\left(\left(x_{n}-T x_{n}\right)+\theta_{n}\left(x_{n}-x_{1}\right)\right)\right\|^{2} \\
\leq & \left\|x_{n}-x_{\delta_{n+1, k}}\right\|^{2}-2 \lambda_{n}\left\langle\left(x_{n}-T x_{n}\right)+\theta_{n}\left(x_{n}-x_{1}\right), j\right\rangle \\
= & \left\|x_{n}-x_{\delta_{n+1, k}}\right\|^{2}-2 \lambda_{n} \theta_{n}\left\|x_{n+1}-x_{\delta_{n+1, k}}\right\|^{2}+2 \lambda_{n}\left\langle\theta_{n}\left(x_{n+1}-x_{n}\right)\right. \\
& -\left(x_{n}-T x_{n}\right)+\theta_{n}\left(x_{1}-x_{\delta_{n+1, k}}\right)+\left(x_{n+1}-T x_{n+1}\right) \\
& \left.-\left(x_{n+1}-T x_{n+1}\right), j\right\rangle .
\end{aligned}
$$

Since $T$ is pseudocontractive, we have for some $j \in J\left(x_{n+1}-x_{\delta_{n+1, k}}\right)$

$$
\begin{aligned}
\left\langle x_{n+1}-T x_{n+1}, j\right\rangle & =\left\langle x_{n+1}-x_{\delta_{n+1, k}}, j\right\rangle-\left\langle T x_{\delta_{n+1, k}}-x_{\delta_{n+1, k}}, j\right\rangle+\left\langle T x_{\delta_{n+1, k}}-T x_{n+1}, j\right\rangle \\
& \geq-\left\|T x_{\delta_{n+1, k}}-x_{\delta_{n+1, k}}\right\| \cdot\left\|x_{n+1}-x_{\delta_{n+1, k}}\right\| \\
& \geq-\delta_{n+1, k}\left\|x_{n+1}-x_{\delta_{n+1, k}}\right\| .
\end{aligned}
$$

Therefore, for such a $j,(16)$ becomes

$$
\begin{aligned}
\left\|x_{n+1}-x_{\delta_{n+1, k}}\right\|^{2} \leq & \left\|x_{n}-x_{\delta_{n+1, k}}\right\|^{2}-2 \lambda_{n} \theta_{n}\left\|x_{n+1}-x_{\delta_{n+1, k}}\right\|^{2}+2 \lambda_{n}\left\langle\theta_{n}\left(x_{n+1}-x_{n}\right)\right. \\
& \left.+\theta_{n}\left(x_{1}-x_{\delta_{n+1, k}}\right)+\left(x_{n+1}-T x_{n+1}\right)-\left(x_{n}-T x_{n}\right), j\right\rangle \\
& +2 \delta_{n+1, k}\left\|x_{n+1}-x_{\delta_{n+1, k}}\right\| \\
\leq & \left\|x_{n}-x_{\delta_{n+1, k}}\right\|^{2}-2 \lambda_{n} \theta_{n}\left\|x_{n+1}-x_{\delta_{n+1, k}}\right\|^{2}+2 \delta_{n+1, k}\left\|x_{n+1}-x_{\delta_{n+1, k}}\right\| \\
& +2 \lambda_{n}\left[(2+L)\left\|x_{n+1}-x_{n}\right\|+\theta_{n}\left\|x_{1}-x_{\delta_{n+1, k}}\right\|\right] \cdot\left\|x_{n+1}-x_{\delta_{n+1, k}}\right\| \\
\leq & \left\|x_{n}-x_{\delta_{n+1, k}}\right\|^{2}-2 \lambda_{n} \theta_{n}\left\|x_{n+1}-x_{\delta_{n+1, k}}\right\|^{2}+2 \delta_{n+1, k}\left\|x_{n+1}-x_{\delta_{n+1, k}}\right\| \\
& +2 \lambda_{n}\left[(2+L) \lambda_{n}\left\|x_{n}-T x_{n}+\theta_{n}\left(x_{n}-x_{1}\right)\right\|+\frac{\theta_{n} r}{2}\right] \cdot\left\|x_{n+1}-x_{\delta_{n+1, k}}\right\| .
\end{aligned}
$$




\section{Moreover}

$$
\begin{aligned}
\left\|x_{n}-T x_{n}+\theta_{n}\left(x_{n}-x_{1}\right)\right\| \leq & \left\|x_{n}-x_{\delta_{n+1, k}}\right\|+\left\|x_{\delta_{n+1, k}}-T x_{\delta_{n+1, k}}\right\| \\
& +\left\|T x_{\delta_{n+1, k}}-T x_{n}\right\|+\left\|x_{n}-x_{\delta_{n+1, k}}\right\|+\theta_{n}\left\|x_{\delta_{n+1, k}}-x_{1}\right\| \\
\leq & (2+L)\left\|x_{n}-x_{\delta_{n+1, k}}\right\|+\delta_{n+1, k}+\frac{\theta_{n} r}{2} .
\end{aligned}
$$

Thus,

$$
\begin{aligned}
\left\|x_{n+1}-x_{\delta_{n+1, k}}\right\|^{2} \leq & \left\|x_{n}-x_{\delta_{n+1, k}}\right\|^{2}-2 \lambda_{n} \theta_{n}\left\|x_{n+1}-x_{\delta_{n+1, k}}\right\|^{2}+2 \delta_{n+1, k}\left\|x_{n+1}-x_{\delta_{n+1, k}}\right\| \\
& +2 \lambda_{n}\left[(2+L) \lambda_{n}\left((2+L)\left\|x_{n}-x_{\delta_{n+1, k}}\right\|+\delta_{n+1, k}+\frac{\theta_{n} r}{2}\right)+\frac{\theta_{n} r}{2}\right] \\
& \cdot\left\|x_{n+1}-x_{\delta_{n+1, k}}\right\| \\
\leq & \left\|x_{n}-x_{\delta_{n+1, k}}\right\|^{2}-2 \lambda_{n} \theta_{n}\left\|x_{n+1}-x_{\delta_{n+1, k}}\right\|^{2}+2 \delta_{n+1, k}\left\|x_{n+1}-x_{\delta_{n+1, k}}\right\| \\
& +2 \lambda_{n}\left[\lambda_{n}(2+L)\left(\left(\frac{5}{2}+L\right)\left(r+2^{-k}\right)+\delta_{n+1, k}\right)+\frac{\theta_{n} r}{2}\right] \cdot\left\|x_{n+1}-x_{\delta_{n+1, k}}\right\| .
\end{aligned}
$$

In the last step we used that by assumption $\left\|x_{n}-x_{\delta_{n+1, k}}\right\| \leq r+2^{-k}$ since $x_{\delta_{n+1, k}}$ a fortiori is a $\delta_{n, k}$-fixed point.

In the same way we have by assumption that $\left\|x_{n+1}-x_{\delta_{n+1, k}}\right\|>\left\|x_{n}-x_{\delta_{n+1, k}}\right\| \geq 0$. In particular, we may divide both sides by $\left\|x_{n+1}-x_{\delta_{n+1, k}}\right\|$. Moreover, $\left\|x_{n+1}-x_{\delta_{n+1, k}}\right\|^{2}-\left\|x_{n}-x_{\delta_{n+1, k}}\right\|^{2}>0$. Hence (17) becomes

$$
2 \lambda_{n} \theta_{n}\left\|x_{n+1}-x_{\delta_{n+1, k}}\right\|<2 \lambda_{n}\left[\lambda_{n}(2+L)\left(\left(\frac{5}{2}+L\right)\left(r+2^{-k}\right)+\delta_{n+1, k}\right)+\frac{\theta_{n} r}{2}\right]+2 \delta_{n+1, k},
$$

and so

$$
\begin{aligned}
\left\|x_{n+1}-x_{\delta_{n+1, k}}\right\| & <\frac{\lambda_{n}}{\theta_{n}}(2+L)\left(\frac{5}{2}+L\right)\left(r+2^{-k}\right)+\frac{r}{2}+\frac{\left(2+2 \lambda_{n}^{2}(2+L)\right) \delta_{n+1, k}}{2 \lambda_{n} \theta_{n}} \\
& \leq \frac{r}{2}+\frac{r+2^{-k}}{2}+\frac{\left(2+2 \lambda_{n}^{2}(2+L)\right) \delta_{n+1, k}}{2 \lambda_{n} \theta_{n}} \leq r+2^{-k-1}+2^{-k-1}=r+2^{-k} .
\end{aligned}
$$

This is a contradiction. Therefore, $\left\|x_{n}-x_{\delta_{n, k}}\right\| \leq r+2^{-k}$ for all $k, n \in \mathbb{N}$.

For $t_{n}:=\frac{1}{1+\theta_{n}}$ we have $\frac{1}{2} \leq t_{n}<1$. By Proposition 1 of [25], we are thus guaranteed the existence of a unique path $\left(y_{t_{n}}\right)_{n \in \mathbb{N}}$ in $K$ such that

$$
y_{t_{n}}=t_{n} T y_{t_{n}}+\left(1-t_{n}\right) x_{1} .
$$

For simplicity we will denote $y_{t_{n}}$ by $y_{n}$. Now we will estimate $\left\|x_{n}-y_{n-1}\right\|$ in such a way that we can apply Lemma 1 to get $\left\|x_{n}-y_{n-1}\right\| \rightarrow 0$ and also a rate of convergence for $\left(\left\|x_{n}-y_{n-1}\right\|\right)_{n}$.

Observe that

$$
\frac{t_{n}}{1-t_{n}}=\frac{1}{1+\theta_{n}} \cdot \frac{1}{1-\frac{1}{1+\theta_{n}}}=\frac{1}{1+\theta_{n}} \cdot \frac{1+\theta_{n}}{\theta_{n}}=\frac{1}{\theta_{n}} .
$$

For all $k, n \in \mathbb{N}$, applying Lemma 2 to $y_{n}$ yields

$$
\begin{aligned}
\left\|y_{n}-x_{\delta_{n+1, k}}\right\| & \leq\left\|x_{1}-x_{\delta_{n+1, k}}\right\|+\frac{t_{n} \delta_{n+1, k}}{1-t_{n}} \\
& \leq b+\frac{\delta_{n+1, k}}{\theta_{n}} \leq \frac{r}{2}+\frac{\delta_{n+1, k}}{\theta_{n}},
\end{aligned}
$$


therefore

$$
\begin{aligned}
\left\|x_{n+1}-y_{n}\right\| & \leq\left\|x_{n+1}-x_{\delta_{n+1, k}}\right\|+\left\|y_{n}-x_{\delta_{n+1, k}}\right\| \\
& \leq r+2^{-k}+\frac{r}{2}+\frac{\delta_{n+1, k}}{\theta_{n}} .
\end{aligned}
$$

Since this fact is true for all $k \in \mathbb{N}$, we get (using that $\lim _{k \rightarrow \infty} \delta_{n+1, k}=0$ )

$$
\left\|x_{n+1}-y_{n}\right\| \leq \frac{3}{2} r
$$

Moreover, for all $k \in \mathbb{N}$,

$$
\begin{aligned}
\left\|x_{n}-T x_{n}+\theta_{n}\left(x_{n}-x_{1}\right)\right\| & \leq\left\|x_{n}-x_{\delta_{n, k}}\right\|+\delta_{n, k}+\left\|T x_{\delta_{n, k}}-T x_{n}\right\|+\theta_{n}\left\|x_{n}-x_{1}\right\| \\
& \leq(1+L)\left(r+2^{-k}\right)+\left\|x_{n}-x_{\delta_{n, k}}\right\|+\underbrace{\left\|x_{\delta_{n, k}}-x_{1}\right\|}_{<b \leq \frac{r}{2}}+\delta_{n, k} \\
& <\left(\frac{5}{2}+L\right)\left(r+2^{-k}\right)+\delta_{n, k} .
\end{aligned}
$$

Hence,

$$
\left\|x_{n}-T x_{n}+\theta_{n}\left(x_{n}-x_{1}\right)\right\| \leq\left(\frac{5}{2}+L\right) r .
$$

For proving (3.5) in the proof of Theorem 3.1 of [8], the hypothesis $F(T) \neq \emptyset$ is not used. Therefore, for all $n \in \mathbb{N}$,

$$
\begin{aligned}
\left\|x_{n+1}-y_{n}\right\|^{2} \leq\left\|x_{n}-y_{n}\right\|^{2}-2 \lambda_{n} \theta_{n} & \left\|x_{n+1}-y_{n}\right\|^{2} \\
& +2 \lambda_{n}^{2}(2+L)\left\|x_{n}-T x_{n}+\theta_{n}\left(x_{n}-x_{1}\right)\right\| \cdot\left\|x_{n+1}-y_{n}\right\| .
\end{aligned}
$$

Using (20) and (21), this becomes

$$
\left\|x_{n+1}-y_{n}\right\|^{2} \leq\left\|x_{n}-y_{n}\right\|^{2}-2 \lambda_{n} \theta_{n}\left\|x_{n+1}-y_{n}\right\|^{2}+3 r^{2} \lambda_{n}^{2}\left(\frac{5}{2}+L\right)(2+L) .
$$

The only term which prevents us from applying Lemma 1 is $\left\|x_{n}-y_{n}\right\|$. In order to resolve this problem, we use Lemma 3 and (19) to obtain

$$
\begin{aligned}
\left\|y_{n}-y_{n-1}\right\| & \leq\left|\frac{\theta_{n-1}}{\theta_{n}}-1\right| \cdot\left\|y_{n-1}-x_{1}\right\| \\
& \leq\left|\frac{\theta_{n-1}}{\theta_{n}}-1\right|\left(\left\|y_{n-1}-x_{\delta_{n, k}}\right\|+\left\|x_{1}-x_{\delta_{n, k}}\right\|\right) \\
& \leq\left|\frac{\theta_{n-1}}{\theta_{n}}-1\right| \cdot\left(2\left\|x_{1}-x_{\delta_{n, k}}\right\|+\frac{\delta_{n, k}}{\theta_{n-1}}\right) \\
& <\left|\frac{\theta_{n-1}}{\theta_{n}}-1\right|\left(2 b+\frac{\delta_{n, k}}{\theta_{n-1}}\right) .
\end{aligned}
$$

This again holds for all $k \in \mathbb{N}$ and so $\left\|y_{n}-y_{n-1}\right\| \leq\left|\frac{\theta_{n-1}}{\theta_{n}}-1\right| 2 b \leq\left|\frac{\theta_{n-1}}{\theta_{n}}-1\right| r$. Therefore, (22) becomes (using also (20))

$$
\begin{aligned}
\underbrace{\left\|x_{n+1}-y_{n}\right\|^{2}}_{a_{n+1}:=} \leq \underbrace{\left\|x_{n}-y_{n-1}\right\|^{2}}_{a_{n}:=} & -\underbrace{2 \lambda_{n} \theta_{n}}_{b_{n}:=}\left\|x_{n+1}-y_{n}\right\|^{2} \\
+ & \underbrace{r^{2}\left|\frac{\theta_{n-1}}{\theta_{n}}-1\right|^{2}+3 r^{2}\left|\frac{\theta_{n-1}}{\theta_{n}}-1\right|+3 \lambda_{n}^{2}\left(\frac{5}{2}+L\right)(2+L) r^{2}}_{c_{n}:=} .
\end{aligned}
$$


We now apply Lemma 1 with

$$
\begin{aligned}
& \psi(x):=x^{2} \\
& N_{1}(\varepsilon):=\max \left\{R_{3}\left(\frac{2 \varepsilon d}{3 r^{2}}\right), R_{4}\left(\sqrt{\frac{\varepsilon}{r^{2}}+\frac{9}{4}}-\frac{3}{2}\right)\right\} \\
& N_{2}(x):=R_{2}\left(\frac{x}{2}\right)+1
\end{aligned}
$$

to obtain a rate of convergence for $\left(a_{n}\right)$. It remains to show that $N_{1}, N_{2}$ as above satisfy conditions (3) and (4) of Lemma 1, respectively. We split the first claim into two parts, namely $(i)$ and $(i i)$ : For all $n \geq N_{1}(\varepsilon)$,

$$
\frac{c_{n}}{b_{n}}=\underbrace{\frac{\frac{3 r^{2}}{2}\left|\frac{\theta_{n-1}}{\theta_{n}}-1\right|}{\lambda_{n} \theta_{n}}+\frac{\frac{r^{2}}{2}\left|\frac{\theta_{n-1}}{\theta_{n}}-1\right|^{2}}{\lambda_{n} \theta_{n}}}_{\substack{(i) \\<\frac{\varepsilon}{2}}}+\underbrace{\frac{3 \lambda_{n}}{2 \theta_{n}}\left(\frac{5}{2}+L\right)(2+L) r^{2}}_{\substack{(i i) \\<\frac{\varepsilon}{2}}} .
$$

Proof of $(i)$. In the following, we will denote $\frac{\left|\frac{\theta_{n}-1}{\theta_{n}}-1\right|}{\lambda_{n} \theta_{n}}$ by $\alpha_{n}$ to simplify notation. By hypothesis, for all $n \geq N_{1}(\varepsilon) \geq R_{4}\left(\sqrt{\frac{\varepsilon}{r^{2}}+\frac{9}{4}}-\frac{3}{2}\right)$ :

$$
\alpha_{n}<\sqrt{\frac{\varepsilon}{r^{2}}+\frac{9}{4}}-\frac{3}{2}
$$

which implies

$$
\left(\alpha_{n}+\frac{3}{2}\right)^{2}<\frac{\varepsilon}{r^{2}}+\frac{9}{4}
$$

and so

$$
r^{2} \alpha_{n}^{2}+3 r^{2} \alpha_{n}<\varepsilon
$$

Since $\lambda_{n} \theta_{n} \leq 1$ we get

$$
\begin{aligned}
\frac{3 r^{2}\left|\frac{\theta_{n-1}}{\theta_{n}}-1\right|}{\lambda_{n} \theta_{n}}+\frac{r^{2}\left|\frac{\theta_{n-1}}{\theta_{n}}-1\right|^{2}}{\lambda_{n} \theta_{n}} \leq \frac{3 r^{2}\left|\frac{\theta_{n-1}}{\theta_{n}}-1\right|}{\lambda_{n} \theta_{n}}+\frac{r^{2}\left|\frac{\theta_{n-1}}{\theta_{n}}-1\right|^{2}}{\left(\lambda_{n} \theta_{n}\right)^{2}} & =3 r^{2} \alpha_{n}+r^{2} \alpha_{n}^{2} \\
& <\varepsilon .
\end{aligned}
$$

Thus implying $(i)$.

Proof of $(i i)$. By hypothesis, $\frac{\lambda_{n}}{\theta_{n}}<\frac{2 \varepsilon d}{3 r^{2}}$ for all $n \geq N_{1}(\varepsilon) \geq R_{3}\left(\frac{\varepsilon d}{r^{2}}\right)$, hence the claim follows by the definition of $b$.

We now show that $N_{2}$ satisfies (4) of Lemma 1 . For all $x \in \mathbb{R}$ the following holds:

$$
\begin{aligned}
\sum_{i=1}^{N_{2}(x)} b_{i} & =2 \sum_{i=1}^{R_{2}\left(\frac{x}{2}\right)+1} \lambda_{i} \theta_{i}>\sum_{i=1}^{R_{2}\left(\frac{x}{2}\right)} \lambda_{i} \theta_{i} \\
& \geq 2 \cdot \frac{x}{2}=x .
\end{aligned}
$$

Therefore, the conditions of Lemma 1 are satisfied and consequently for $\Phi$ as in Lemma 1 ,

$$
\forall \delta>0 \forall n \geq \Phi\left(N_{1}, N_{2}, \delta\right)\left(\left\|x_{n}-y_{n-1}\right\|<\delta\right) .
$$

Remark 2. The authors of this paper acknowledge that the proof of the induction step is almost exactly as in Chidume and Zegeye [8]; we only needed to make some minor adjustments to accommodate for the fact that the hypothesis $F(T) \neq \emptyset$ was weakened. 
Finally, we proceed to showing the actual statement of the Theorem.

It follows from Lemma 1 with $\delta=\frac{\varepsilon}{2(1+L)}$ that $\tilde{N}=N_{1}\left(\frac{\varepsilon^{2}}{8(1+L)^{2}}\right)=N_{1}\left(\frac{\psi\left(\frac{\varepsilon}{2(1+L)}\right)}{2}\right)$ and

$$
\begin{aligned}
C & =\frac{18(1+L)^{2} r^{2}}{\varepsilon^{2}}+2(\tilde{N}-1) \\
& \geq \frac{8(1+L)^{2} a_{\tilde{N}}^{2}}{\varepsilon^{2}}+\sum_{n=1}^{\tilde{N}-1} b_{n} \\
& =\frac{2 a_{\tilde{N}}^{2}}{\psi\left(\frac{\varepsilon}{2(1+L)}\right)}+\sum_{n=1}^{\tilde{N}-1} b_{n}
\end{aligned}
$$

since $a_{n}=\left\|x_{n}-y_{n-1}\right\| \leq \frac{3 r}{2}$ for all $n \in \mathbb{N}$ and so, in particular, $a_{\tilde{N}}^{2} \leq \frac{9 r^{2}}{4}$. So, in particular, for all natural numbers $n$ such that $n \geq \Psi\left(b, L, R_{1}, R_{2}, R_{3}, R_{4}, \varepsilon\right) \geq N_{2}^{N}(C)+1$ we have

$$
\left\|x_{n}-y_{n-1}\right\| \leq \frac{\varepsilon}{2(1+L)} .
$$

Moreover, $\left\|y_{n}-T y_{n}\right\|=\left\|t_{n} T y_{n}+\left(1-t_{n}\right) x_{1}-T y_{n}\right\|$ for all $n \in \mathbb{N}$ by the definition of $y_{n}$. Thus, from (18)

$$
\begin{aligned}
\left\|x_{n}-T x_{n}\right\| & \leq\left\|x_{n}-y_{n-1}\right\|+\left\|y_{n-1}-T y_{n-1}\right\|+\left\|T y_{n-1}-T x_{n}\right\| \\
& \leq(1+L)\left\|x_{n}-y_{n-1}\right\|+\left(1-t_{n-1}\right)\left\|T y_{n-1}-x_{1}\right\|,
\end{aligned}
$$

where (using that $t_{n-1} \geq \frac{1}{2}$ )

$$
\begin{aligned}
\left\|T y_{n-1}-x_{1}\right\| & =\left\|\frac{y_{n-1}-\left(1-t_{n-1}\right) x_{1}}{t_{n-1}}-x_{1}\right\| \\
& =\left\|\frac{y_{n-1}-x_{1}}{t_{n-1}}\right\| \\
& \leq 2 r .
\end{aligned}
$$

In the last step we have used that $\left\|y_{n-1}-x_{1}\right\| \leq r$ which holds since

$$
\left\|y_{n-1}-x_{1}\right\| \leq\left\|y_{n-1}-x_{\delta_{n, k}}\right\|+\left\|x_{\delta_{n, k}}-x_{1}\right\| \leq b+\frac{\delta_{n, k}}{\theta_{n-1}}+b
$$

for all $k$.

Since furthermore $1-t_{n-1}=\frac{\theta_{n-1}}{1+\theta_{n-1}}<\theta_{n-1}<\frac{\varepsilon}{4 r}$ for all $n \geq \Psi\left(b, L, R_{1}, R_{2}, R_{3}, R_{4}, \varepsilon\right) \geq$ $R_{1}\left(\frac{\varepsilon}{4 r}\right)+1,(24)$ implies

$$
\begin{aligned}
\left\|x_{n}-T x_{n}\right\| & \leq(1+L)\left\|x_{n}-y_{n-1}\right\|+\left(1-t_{n-1}\right)\left\|T y_{n-1}-x_{1}\right\| \\
& <\frac{\varepsilon}{2}+\frac{\varepsilon}{2}=\varepsilon
\end{aligned}
$$

and the proof is complete.

We remark that in the above proof, the hypothesis that $T$ has approximate fixed points $x_{\delta}$, whose distance to the starting point $x_{1}$ of the iteration sequence is smaller than $b$, was only used to construct bounds on $\left(x_{n}\right)$ and $\left(y_{n}\right)$. Since both $\left(x_{n}\right) \subset K$ and $\left(y_{n}\right) \subset K$, we can instead assume that $K$ is bounded with diameter $\operatorname{diam}(K):=\sup \{\|x-y\|: x, y \in K\} \leq M$ for some $M \in \mathbb{R}_{+}^{*}$. In this case we get a rate of convergence which depends on $M$ instead of $b$. 
Corollary 1. Let $K$ be a nonempty, closed, convex and bounded subset of a real Banach space $X$ with diam $(K) \leq M$ for some $M \in \mathbb{R}$. Let $T: K \rightarrow K$ be a Lipschitz pseudocontractive mapping with Lipschitz constant L. Let $\left(x_{n}\right)$ be the sequence generated from an arbitrary $x_{1} \in K$ by

$$
x_{n+1}:=\left(1-\lambda_{n}\right) x_{n}+\lambda_{n} T x_{n}-\lambda_{n} \theta_{n}\left(x_{n}-x_{1}\right)
$$

Given rates of convergence/divergence $R_{i}: \mathbb{R} \rightarrow \mathbb{N}$ satisfying (i)' to (iv)' above, we get

$$
\forall \varepsilon>0 \forall n \geq \Psi\left(M, L, R_{1}, R_{2}, R_{3}, R_{4}, \varepsilon\right)\left(\left\|x_{n}-T x_{n}\right\|<\varepsilon\right)
$$

where

$$
\Psi\left(M, L, R_{1}, R_{2}, R_{3}, R_{4}, \varepsilon\right)=\max \left\{N_{2}(C)+1, R_{1}\left(\frac{\varepsilon}{2 M}\right)+1\right\}
$$

and

$$
\begin{aligned}
& N_{1}(\varepsilon):=\max \left\{R_{3}\left(\frac{\varepsilon}{4 M^{2}(2+L)}\right), R_{4}\left(\sqrt{\frac{\varepsilon}{M^{2}}+1}-1\right)\right\}, \\
& N_{2}(x):=R_{2}\left(\frac{x}{2}\right)+1, \\
& C:=\frac{8(1+L)^{2} M^{2}}{\varepsilon^{2}}+2\left(N_{1}\left(\frac{\varepsilon^{2}}{8(1+L)^{2}}\right)-1\right) .
\end{aligned}
$$

Proof. The proof follows with the same arguments as the proof of Theorem 1 but becomes much simpler since finding bounds for $\left\|x_{n}-y_{n}\right\|$ as in Theorem 1 is now trivial. For more details see $[15]$.

Remark 3. The complexity of the modulus of asymptotic regularity is strongly reduced in Corollary 1. While it is dependent on $(2+L)^{R_{3}\left(L^{2}\right)^{2}}$ in Theorem 1, the modulus provided in Corollary 1 exhibits only polynomial growth in its arguments. This is due to the fact that for the extraction of the bound of the iteration sequence $x_{n}$, we needed some crude estimates, while this is trivial when we assume that $K$ is bounded.

\section{Examples of Sequences $\lambda_{n}, \theta_{n}$}

We now give examples of sequences $\lambda_{n}$ and $\theta_{n}$ which satisfy conditions (i) to (v) above, taken from [8]. We then calculate the rates of convergence and divergence $R_{i}: \mathbb{R} \rightarrow \mathbb{N}$ and substitute them into the functional $\Psi$ in Theorem 1 . Let $a, b \in \mathbb{R}$ with $0<a<b$ and $a+b<1$. Define

$$
\lambda_{n}:=\frac{1}{(n+1)^{a}} \quad \text { and } \quad \theta_{n}:=\frac{1}{(n+1)^{b}} .
$$

If $R_{1}(\varepsilon):=\left\lceil\varepsilon^{-\frac{1}{b}}-1\right\rceil$, we get $\forall \varepsilon>0 \forall n \geq R_{1}(\varepsilon)\left(\theta_{n}=\frac{1}{(n+1)^{b}} \leq \varepsilon\right)$.

In order to simplify notation we denote $\lambda_{n} \theta_{n}$ by $d_{n}$ and define

$$
\begin{aligned}
& R_{2}(h):=\left\lceil\sum_{n=1}^{\tilde{h}} 2^{n-1}\right\rceil, \text { where } \\
& \tilde{h}:=\left\lceil\frac{\log _{2}\left(\frac{h\left(2^{1-a-b}-1\right)}{2^{-a-b}}+1\right)}{1-a-b}\right] .
\end{aligned}
$$

We will show that $R_{2}$ is a rate of divergence of $d_{n}$. Observe that $d_{n} \geq \frac{1}{n+1}$ for all $n \in \mathbb{N}$, so we could take the much simpler term $2^{\lceil h\rceil+1}$, which is the 'textbook' rate of divergence of the harmonic series. We now show that $R_{2}$ is as required. 
When considering the sum $\sum_{n=1}^{R_{2}(h)} d_{n}$ for $h \geq 0$, we see that we sum up $\tilde{h}$-many "packets", where the $n$th packet has $2^{n-1}$ summands. We denote the $n$th packet by $p_{n}$ :

$$
p_{n}:=d_{2^{n-1}}+\ldots+d_{2^{n}-1} .
$$

Thus, we get

$$
\sum_{n=1}^{R_{2}(h)} d_{n}=\sum_{n=1}^{\tilde{h}} p_{n} .
$$

Since $d_{n}$ is strictly decreasing, we get

$$
p_{n}>\underbrace{2^{n-1}}_{\text {number of summands }} \cdot \underbrace{2^{-n(a+b)}}_{d_{2^{n}-1}} .
$$

Hence (25) becomes

$$
\begin{aligned}
\sum_{n=1}^{R_{2}(h)} d_{n} & >\sum_{n=1}^{\tilde{h}} 2^{n-1-n(a+b)} \\
& =\frac{1}{2} \sum_{l=1}^{\tilde{h}} 2^{n(1-a-b)} \\
& =\frac{1}{2} \cdot \frac{2^{1-a-b}}{2^{1-a-b}-1}\left(2^{\tilde{h}(1-a-b)}-1\right) \\
& \geq \frac{2^{-a-b}}{2^{1-a-b}-1} \cdot \frac{\left(2^{1-a-b}-1\right) h}{2^{-a-b}} \\
& =h .
\end{aligned}
$$

If $R_{3}(\varepsilon):=\left\lceil\varepsilon^{\frac{1}{a-b}}-1\right\rceil$, we get $\forall \varepsilon>0 \forall n \geq R_{3}(\varepsilon)\left(\frac{\lambda_{n}}{\theta_{n}}=\frac{1}{(n+1)^{b-a}} \leq \varepsilon\right)$. get

We define $R_{4}(\varepsilon):=\left\lceil\left(\frac{\varepsilon}{2^{b}(a+b)}\right)^{\frac{1}{a+b-1}}-1\right\rceil$ and then for all $\varepsilon>0$ and for each $n \geq R_{4}(\varepsilon)$, we

$$
\begin{aligned}
\frac{\frac{\theta_{n-1}}{\theta_{n}}-1}{\lambda_{n} \theta_{n}} & =\left(\left(\frac{n+1}{n}\right)^{b}-1\right)(n+1)^{a+b} \\
& \leq\left(\frac{(n+1)^{b}-n^{b}}{n^{b}}\right) 2^{b} n^{b}(n+1)^{a} \\
& <2^{b}\left((n+1)^{a+b}-n^{a+b}\right)
\end{aligned}
$$

By the mean value theorem, there exists $\xi \in(n, n+1)$ such that $(n+1)^{a+b}-n^{a+b}=(a+b) \xi^{a+b-1}$ and hence

$$
2^{b}\left((n+1)^{a+b}-n^{a+b}\right)<2^{b}(a+b)(n+1)^{a+b-1} .
$$

Thus (26) implies $\frac{\frac{\theta_{n-1}}{\theta_{n}}-1}{\lambda_{n} \theta_{n}}<\varepsilon$ and $R_{4}$ is the desired rate of convergence.

\section{References}

[1] BAillon, J., AND BRUCK, R.E. The rate of asymptotic regularity is $0\left(\frac{1}{\sqrt{n}}\right)$. Theory and applications of nonlinear mappings of accretive and monotone type, Lecture Notes in Pure and Appl. Math. 178, pp. 51-81, Dekker, New York, 1996. 
[2] Borwein, J., Reich, S., And Shafrir, I. Krasnoselski-Mann iterations in normed spaces. Canad. Math. Bull. 35, (1992), pp. 21-28.

[3] Browder, F.E. Nonlinear mappings of nonexpansive and accretive type in Banach spaces. Bulletin of the American Mathematical Society 73, (1967), pp. 875-882.

[4] Browder, F.E., And Petryshyn, W.V. Constructions of fixed points of nonlinear mappings in Hilbert space. Journal of Mathematical Analysis and Applications 20, (1967), pp. 197-228.

[5] BRUCK, R.E. A strongly convergent iterative method for the solution of $0 \in U(x)$ for a maximal monotone operator $U$ in Hilbert space. Journal of Mathematical Analysis and Applications 48, (1974), pp. 114-126.

[6] Chidume, C.E. Geometric Properties of Banach Spaces and Nonlinear Iterations. Springer Lecture Notes in Mathematics vol. 1965, xi+326pp., (2009).

[7] Chidume, C.E., And Mutangadura, S.A. An example on the Mann iteration method for Lipschitz pseudocontractions. Proceedings of the American Mathematical Society 129, (2001), pp. 2359-2363.

[8] Chidume, C. E., And Zegeye, H. Approximate fixed point sequences and convergence theorems for Lipschitz pseudocontractive maps. Proceedings of the American Mathematical Society 132, 3 (2004), pp. 831-840.

[9] Gerhardy, P., and Kohlenbach, U. Strongly uniform bounds from semi-constructive proofs. Annal of Pure and Applied Logic 141, (2006), pp. 89-107.

[10] Gerhardy, P., and Kohlenbach, U. General logical metatheorems for functional analysis. Transactions of the American Mathematical Society 360, (2008), pp. 2615-2660.

[11] Groetsch, C.W. A note on segmenting Mann iterates. Journal of Mathematical Analysis and Applications 40, (1972), pp. 369-372.

[12] Ishikawa, S. Fixed points by a new iteration method. Proceedings of the American Mathematical Society 44, (1974), pp. 147-150.

[13] IshikawA, S. Fixed points and iterations of a nonexpansive mapping in a Banach space. Proceedings of the American Mathematical Society 59, (1976), pp. 65-71.

[14] Kato, T. Nonlinear semigroups and evolution equations. Journal of the Mathematical Society of Japan 19, (1967), pp.508-520.

[15] Körnlein, D. Logical Extraction of Bounds on Asymptotic Regularity for Pseudocontractions. Bachelor Thesis, 31pp., TU Darmstadt 2010.

[16] Kohlenbach, U. On the computational content of the Krasnoselski and Ishikawa fixed point theorems. In: Proceedings of the Fourth Workshop on Computability and Complexity in Analysis, J. Blanck, V. Brattka, P. Hertling (eds.), Springer LNCS 2064, (2001), pp. 119-145.

[17] Kohlenbach, U. A quantitative version of a theorem due to Borwein-Reich-Shafrir. Numerical Functional Analysis and Optimization 22, (2001), pp. 641-656.

[18] Kohlenbach, U. Uniform asymptotic regularity for Mann iterates. Journal of Mathematical Analysis and Applications 279, (2003), pp. 531-544.

[19] Kohlenbach, U. Some logical metatheorems with applications in functional analysis. Transactions of the American Mathematical Society 357, 1 (2005), pp. 89-128.

[20] Kohlenbach, U. Applied Proof Theory: Proof Interpretations and their Use in Mathematics. Springer Monographs in Mathematics. xx+536pp., Springer Heidelberg-Berlin, 2008. 
[21] Kohlenbach, U., And Leustean, L. Mann iterates of directionally nonexpansive mappings in hyperbolic spaces. Abstract and Applied Analysis 2003, 8 (2003), pp. 449-477.

[22] Kohlenbach, U., and Leuştean, L. Asymptotically nonexpansive mappings in uniformly convex hyperbolic spaces. Journal of the European Mathematical Society 12, (2010), pp. 71-92.

[23] Krasnoselski, M. A. Two remarks on the method of successive approximation. Usp. Math. Nauk (N.S.) 10, (1955), pp. 123-127 (Russian).

[24] Moore, C., ANd Nnoli, B. V. C. Iterative solution of nonlinear equations involving setvalued uniformly accretive operators. Computers \&3 Mathematics with Applications 42, 1-2 (2001), pp. 131-140.

[25] Morales, C., And Jung, J. S. Convergence of paths for pseudo-contractive mappings in Banach spaces. Proceedings of the American Mathematical Society 128 (2000), pp. 3411-3419.

[26] Osilike, M.O., and Udomene, A. Demiclosedness Principle and convergence theorems for strictly pseudocontractive mappings of Browder-Petryshyn type. Journal of Mathematical Analysis and Applications 256, (2001), pp. 431-445.

[27] Petryshyn, W.V. A characterization of strict convexity of Banach spaces and other uses of duality mappings. Journal of Functional Analysis 6, (1970), pp. 282-291.

[28] Reich, S. Extension problems for accretive sets in Banach space. Journal of Functional Analysis 26, (1977), pp. 378-395.

[29] Reich, S. Iterative mathods for accretive set. In: Nonlinear Equations in Abstract Spaces, Academic Press, New York 1978, pp. 317-326.

[30] ReICH, S. Weak convergence theorems for nonexpansive mappings in Banach spaces. Journal of Mathematical Analysis and Applications 67, (1979), pp. 274-276.

[31] Reich, S. Strong convergence theorems for resolvents of accretive operators in Banach spaces. Journal of Mathematical Analysis and Applications 75, (1980), pp. 287-292.

[32] TAO, T. Soft analysis, hard analysis, and the finite convergence principle. Essay posted May 23, 2007. Appeared in: T. Tao, Structure and Randomness: Pages from Year One of a Mathematical Blog, AMS, 298pp., 2008. 